Preprints of the

Max Planck Institute for

Research on Collective Goods

Bonn 2011/11

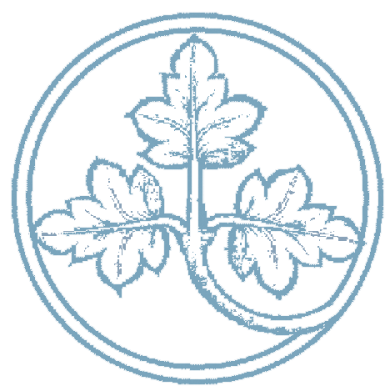

Pareto-Optimal

Assignments by

Hierarchical Exchange

Sophie Bade

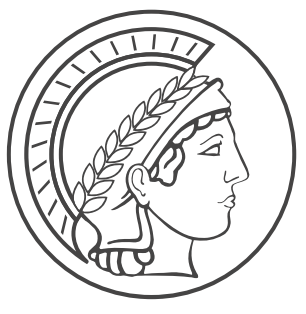




\section{Pareto-Optimal Assignments by Hierarchical Exchange}

Sophie Bade

June 2011 


\title{
Pareto-Optimal Assignments by Hierarchical Exchange
}

\author{
SOPHIE BADE*广
}

3rd June 2011

\begin{abstract}
A version of the Second Fundamental Theorem of Welfare Economics that applies to a money-free environment, in which a set of indivisible goods needs to be matched to some set of agents, is established. In such environments, "trade" can be identified with the set of hierarchical exchange mechanisms defined by Papai (2000). Papai (2000)'s result - that any such mechanism yields Pareto-optimal allocations - can be interpreted as a version of the First Fundamental Theorem of Welfare Economics for the given environment. In this note, I show that for any Pareto-optimal allocation and any hierarchical exchange mechanism one can find an initial allocation of ownership rights, such that the given Pareto-optimal allocation arises as a result of trade.
\end{abstract}

*Max Planck Institute for Research on Collective Goods, Kurt-Schumacher-Str. 10, D-53113 Bonn, Germany. Phone: 49+228+9141670 Fax: 49+228+9141670

†I would like to thank Martin Hellwig and Anne-Katrin Roesler for their comments. 
The First Fundamental Theorem of Welfare Economics provides conditions under which every equilibrium of competitive markets is Paretooptimal. The Second Fundamental Theorem gives some conditions under which every Pareto-optimal allocation can be sustained as a competitive equilibrium. Both theorems apply to markets with divisible goods and money. The present note is concerned with an analogue of the Second Fundamental Theorem of Welfare Economics for a money-free environment in which some indivisible goods need to be matched to agents.

The problems considered here are known as "housing problems". A housing problem $(N, K, R)$ consists in two finite sets of objects, henceforth called houses, and of agents, $K$ and $N$, respectively, and $R$ : $=\left(R_{1}, \cdots, R_{n}\right)$ a profile of (strict) preferences of all agents over the houses. The partial profiles of the preferences of the agents in $M \subset N$ and the remainder are denoted by $R_{M}, R_{-M}$, respectively. Agent $i$ 's most preferred element in $K$ according to $R_{i}$ is denoted by top $\left(R_{i}\right)$. Finally, as usual $P_{i}$ denotes the strict part of $R_{i}$.

An allocation defined as any injective function from the smaller set of $K$ and $N$ to the larger one. In terms of substance, an allocation is such that no agent obtains more than one house, some agents do not obtain houses if and only if there are more agents than houses $(|N|>|K|)$, and some houses remain unmatched if and only if there are more houses than agents $(|K|>|N|)$. An allocation is denoted as a vector $x:=\left(x_{1}, \cdots, x_{|N|}\right)$ with the understanding that $x_{i}$ denotes the house matched to agent $i$, where $x_{i}=\emptyset$ denotes the case in which $i$ does not obtain a house for the given allocation. Agents are assumed to care only about their own matches and to prefer any house to no house. As a shorthand, preferences over allocations are also denoted by $R_{i}$ with the understanding that $x R x^{\prime}$ holds if and only if $x_{i} R_{i} x_{i}^{\prime}$.

Any function from $K$ to $N$ is called an assignment. According to an assignment, multiple houses might be mapped to the same agent. If $|N| \geq \mid$ $K \mid$ the set of allocations is a subset of the set of assignments. Otherwise allocations are such that some houses remain unmatched, which is ruled out for assignments.

Let $\mathcal{R}$ be the set of all profiles $R$. A social-choice function $f$ maps any profile $R \in \mathcal{R}$ to an allocation $f(R)$. The houses that are matched to the 
agents in $M$ under allocation $f(R)$ are denoted by $f_{M}(R)$. A mechanism $\Gamma$ is a direct revelation mechanism if the agents' strategies consist in directly revelations of rankings over the available houses to the designer. The outcome of direct revelation mechanism $\Gamma$ when agents claim the preference profile $R$ is denoted as $\Gamma(R)$, the social choice rule that maps any profile of preferences $R \in \mathcal{R}$ to $\Gamma(R)$ is denoted by $f^{\Gamma}$. If the rule $f^{\Gamma}$ satisfies some property $X$, then the mechanism $\Gamma$ is said to satisfy this property $X$.

To develop an analogue of the Second Fundamental Theorem of Welfare Economics, a definition of "trade" that is appropriate for the present environment is needed. Papai (2000)'s hierarchical exchange mechanisms provide a natural candidate for this purpose. A hierarchical exchange mechanism specifies a system of ownership rights and determines allocations as the outcomes of free trade for the given ownership rights. The system of ownership rights consists in an initial assignment of all houses to the agents and a set of inheritance rules for the houses. Ownership rights in hierarchical exchange mechanisms are such that each house is always owned by someone. The inheritance rule solves the problem of the ownership of multiple houses before the termination of the mechanism: observe that there is a tension between the following two requirements. On the one hand, the class of trading mechanisms should be general and therefore allow for a wide variety of initial ownership structures, which should in particular comprise the case that some agent starts out owning multiple houses. On the other hand, the mechanism needs to result in an allocation, meaning that no agent is allowed to obtain more than one house. Papai (2000) elegantly solves this problem with the definition of inheritance rules that prescribe how the houses whose owners leave the market should be distributed to the remaining agents. In hierarchical exchange mechanisms, trade is identified with a sequence of trading cycles.

Serial dictatorships and Gale's top trading cycles mechanism are special cases of hierarchical exchange mechanisms (for housing problems with $|K|=$ $|N|)$. In the case of a serial dictatorship, some agent starts out owning all houses. His appropriation of one of the houses can be interpreted as the formation of a trading cycle of length one. The inheritance rule then prescribes that a next agent inherits the entire remainder of houses. In 
Gale's top trading cycles mechanism, each agent starts out owning exactly one house. All agents are then asked to point to the owners of their most preferred house. At least one cycle forms. All agents in this cycle trade houses. The remaining agents repeat the procedure with the set of remaining houses. Inheritance rules do not play any role in Gale's top trading cycles mechanism, as there are no owners of multiple houses.

Formally, Papai (2000) defines hierarchical exchange mechanisms as lists of inheritance trees $\Gamma=\left(\Gamma_{a}(V, Q, \mathcal{L}, \mathcal{H})\right)_{a \in K}$. Each inheritance tree $\Gamma_{a}(V, Q, \mathcal{L}, \mathcal{H})^{1}$ is a rooted tree where $V$ is the set of vertices, and $Q \subset V \times V$ is the set of arcs. The function $\mathcal{L}: V \rightarrow N$ labels each vertex with an individual, the function $\mathcal{H}: Q \rightarrow K$ labels each arc with a house. The root of the tree $\Gamma_{a}$ is labeled with the initial owner of $a$, say this is agent $i$. The labels on the arcs following this vertex denote agent $i$ 's possible matches. If agent $i$ is matched to house $b$, then the label of vertex following on the arc with the label $b$ determines the next owner of $a$. Continuing in this way, one can determine all the potential inheritances by following the appropriate arcs. ${ }^{2}$

To qualify as inheritance trees, the trees have to satisfy a set of technical conditions specified by Papai (2000, p. 1409). These conditions ensure, for example, that no agent appears twice on the same inheritance path. Since the present note does not make explicit use of these conditions, they are not stated here. Hierarchical exchange mechanisms are group-strategyproof in the sense that for all $R$, there do not exist $M \subset N$ and $\widetilde{R}_{M}$ such that $f^{\Gamma}\left(\widetilde{R}_{M}, R_{-M}\right) R_{M} f^{\Gamma}(R)$ and $f_{M}^{\Gamma}\left(\widetilde{R}, R_{-M}\right)_{M} \neq f_{M}^{\Gamma}(R)$. The fact is established as part of the main characterization result in Papai (2000, p. 1425). A mech-

\footnotetext{
${ }^{1}$ I replace Papai (2000)'s definition of an inheritance tree $\Gamma_{a}(V, Q)$ which presumes the labeling functions $\mathcal{L}, \mathcal{H}$ by an expression that explicitly lists the labeling functions: $\Gamma_{a}(V, Q, \mathcal{L}, \mathcal{H})$. This allows me to define the concept of permutations of roles for hierarchical exchange mechanisms.

${ }^{2}$ In terms of this formalism, Gale's top trading cycles mechanisms can be defined through any list of inheritance trees $\Gamma=\left(\Gamma_{a}(V, Q, \mathcal{L}, \mathcal{H})\right)_{a \in K}$ with the feature that no two roots are labeled with the same agent. This implies that each agent starts out owning exactly one house. The inheritance rule consequently plays no role, which in turn implies that any list of inheritance trees with the named feature defines the same to trading cycles mechanisms. Conversely, the root of every tree of a serial dictatorship mechanism is labeled with the same agent. Every vertex ensuing on the initial node is labeled with the second dictator and so forth.
} 
anism is considered strategyproof if the condition of group-strategyproofness holds for all singleton "groups" $M$. A mechanism is called Pareto-optimal if any outcome $x=\Gamma(R)$ of the mechanism is Pareto-optimal according to the profile of preferences $R$. The sets of agents and houses eliminated in the $t$ th round of the mechanism are denoted by $W_{t}(R)$ and $F_{t}(R)$, respectively.

The outcome of a hierarchical exchange mechanism can be calculated sequentially as follows: first, eliminate all agents and houses in the first set of top trading cycles $W_{1}(R)$ and $F_{1}(R)$; their matches are $f_{W_{1}(R)}^{\Gamma}(R)$. The allocation for all other agents $N \backslash W_{1}(R)$ can be calculated as the outcome of a submechanism $\Gamma^{\prime}$, which is defined through a set of inheritance trees $\times_{a \in K^{\prime}} \Gamma_{a}^{\prime}\left(Q^{\prime}, V^{\prime}, \mathcal{L}^{\prime}, \mathcal{H}^{\prime}\right)$ that have been reduced as follows. Let $v_{i}$ be a vertex such that $\mathcal{L}\left(v_{i}\right) \in W_{1}(R)$ and let $a$ be the match of $\mathcal{L}\left(v_{i}\right)$ under the first set of top trading cycles, let $\mathcal{H}\left(v_{i}, v_{j}\right)=a$, in words, let $\left(v_{i}, v_{j}\right)$ be the arc following $v_{i}$ that is labeled with $\mathcal{L}\left(v_{i}\right)$ 's match. Replace $v_{i}$ and the corresponding subtree by the $v_{j}$ and the following subtree (keeping the labels of the latter subtree). The rationale behind this step in the construction of the inheritance trees $\Gamma_{a}^{\prime}\left(Q^{\prime}, V^{\prime}, \mathcal{L}^{\prime}, \mathcal{H}^{\prime}\right)$ is that agents in $W_{1}(R)$ have already been matched to houses and therefore can no longer inherit any houses. Whenever they would inherit a house according to some inheritance tree, that is, whenever their name would appear as the label on some vertex $v_{i}$ in one of the remaining trees, the house that they would inherit is passed on to the agent that follows on the arc labeled that agent's match. Once there is no more vertex for an agent in $W_{1}(R)$ left, go on to eliminate all arcs that are labeled with houses in $F_{1}(R)$ and their ensuing subtrees. This latter step can be explained through the observation that the case that some agent not in $W_{1}(R)$ is matched to a house in $F_{1}(R)$ is irrelevant; these houses have all been already matched in the first round. It is a purely technical matter to show that a submechanism $\Gamma^{\prime}$ satisfies the defining properties for hierarchical exchange mechanism given by Papai (2000, p. 1409).

With this definition of hierarchical exchange mechanisms in hand, one can now state an analogue for the First Fundamental Theorem of Welfare Economics as

Theorem 1 Any hierarchical exchange mechanism $\Gamma=\left(\Gamma_{a}(V, Q, \mathcal{L}, \mathcal{H})\right)_{a \in K}$ is strategyproof and Pareto-optimal. 
Proof Theorem 1 is a part of the main result of Papai (2000, p. 1425) and proven by her.

The main result of the present note is, in a sense, the converse of Theorem 1. In tune with the Second Fundamental Theorem of Welfare Economics, I claim that for any Pareto-optimal allocation $x$ in a housing problem $(N, K, R)$ and any structure of ownership given by some hierarchical exchange mechanisms one can find a way to assign the given ownership rights to the agents such that $x$ is the outcome of the mechanism. To formalize this idea, we need to distinguish between the ownership structure defined through a mechanism and the initial assignment of ownership rights for a given hierarchical exchange mechanism $\Gamma=\left(\Gamma_{a}(V, Q, \mathcal{L}, \mathcal{H})\right)_{a \in K}$. I view the trees together with the labels denoting houses $\left(\Gamma=\left(\Gamma_{a}(V, Q, \cdot \mathcal{H})\right)_{a \in K}\right)$ as the given structure of ownership. The labeling function $\mathcal{L}$ represents a particular assignment defined by the mechanism. Any allocation of initial ownership in a hierarchical exchange mechanism can be achieved through a permutation of the roles played by the agents.

Definition 1 A permutation of roles $p$ consists of a bijection $p: N \rightarrow$ $N$. A permutation of roles $p$ induces a permuted hierarchical exchange mechanism $p(\Gamma)$ which is defined through $p(\Gamma)=\times_{a \in K} \Gamma_{a}(V, Q, p \circ \mathcal{L}, \mathcal{H})$.

A mechanism and its permutation foresee the same types of ownership rights over houses. The only differences between a hierarchical exchange mechanism $\Gamma$ and a permutation of the mechanism $p(\Gamma)$ lies in the permutation of the roles played by the agents. The main result of the note can now be stated as:

Theorem 2 Let $x$ be a Pareto-optimal allocation in a housing problem $(N, K, R)$. Fix a hierarchical exchange mechanism $\Gamma$. Then there exists a permutation of roles $p$ such that $x$ is the outcome of $p(\Gamma)$.

The proof of this result is based on the idea that in each Pareto optimum there is some agent $i$ who obtains his most preferred house out of the grand set $\operatorname{top}\left(R_{i}\right)$. If the initial assignment is such that this agent starts out owning this house, he will form a trading cycle of length one by pointing to himself, 
leaving the mechanism. Since $x$ is Pareto-optimal, there exists an agent $j$ who is matched to his most preferred house $x_{j}$ out of the remaining houses. Ownership rights on the remainder of houses are arranged such that, after the elimination of the first agent, this agent $j$ becomes the owner of $x_{j}$. The proof proceeds inductively until all ownership rights have been determined. To make this proof work, I first need to show that the outcome of a hierarchical exchange mechanism does not depend on the order in which top trading cycles are eliminated. (According to Papai (2000)'s definition all cycles that form in some stage $t$ eliminated simultaneously.) I do so in the next two lemmata.

Definition 2 An assignment rule $f$ is group-nonbossy if for all $R \subset \mathcal{R}$, $M \subset N$, and $\widetilde{R}_{M}, f_{M}(R)=f_{M}\left(\widetilde{R}_{M}, R_{-M}\right)$ implies $f(R)=f\left(\widetilde{R}_{M}, R_{-M}\right)$.

Group-nonbossiness ensures that groups cannot change the assignment for agents that do not belong to the group, by reporting different preferences without changing their own. If one restricts the groups $M$ in the definition to singletons, one obtains the standard definition of nonbossiness (as defined, for example, by Papai (2000, p. 1422)).

Lemma 1 Any hierarchical exchange mechanism is group-nonbossy.

Proof Suppose there existed a profile of preferences $R$, a hierarchical exchange mechanism $\Gamma$, a subset $M \subset N$, and $\widetilde{R}_{M}$ such that $f_{M}^{\Gamma}(R)=f_{M}^{\Gamma}\left(\widetilde{R}_{M}, R_{-M}\right)$ holds, but $f^{\Gamma}(R) \neq f^{\Gamma}\left(\widetilde{R}_{M}, R_{-M}\right)$. The latter implies that there is some $j \notin$ $M$ such that $f_{j}^{\Gamma}(R) \neq f_{j}^{\Gamma}\left(\widetilde{R}_{M}, R_{-M}\right)$. Now consider the group $M^{\prime}=M \cup\{j\}$. First, consider the case in which $f_{j}^{\Gamma}\left(\widetilde{R}_{M}, R_{-M}\right) P_{j} f_{j}^{\Gamma}(R)$. Then the hierarchical exchange mechanism is not group-strategyproof, as the agents in $M^{\prime}$ can improve the utility of one of its members (namely $j$ ), while keeping the utility of all other members constant through misrepresenting their preferences ( $\widetilde{R}_{M}$ for the members of $M$ and the truthful $R_{j}$ for agent $j$ ). If, on the other hand, $f_{j}^{\Gamma}(R) P_{i} f_{j}^{\Gamma}\left(\widetilde{R}_{M^{\prime}}, R_{-M^{\prime}}\right)$, the same argument holds true for the same group if the true preferences of agents are $\left(\widetilde{R}_{M}, R_{-M}\right)$ and the agents in $M$ misrepresent them to be $R_{M}$. This yields a contradiction with the characterization result proved by Papai (2000, p. 1425), which states that hierarchical exchange rules are group-strategyproof. 
Lemma 2 The order of elimination of top trading cycles does not matter.

Proof Suppose there exists a mechanism $\Gamma$ and some preference profile $R$, such that $\Gamma(R)$ eliminates several cycles at some stage, say $t$. Suppose in that stage there are two groups of cycles involving the agents in $N_{1}$ and $N_{2}$ so $N_{1} \cup N_{2}=W_{t}(R)$, and suppose that the allocation following the hierarchical exchange rule, as defined by Papai (2000) $f^{\Gamma}(R)$, differs from the allocation when the cycle(s) involving the agents in $N_{1}$ are eliminated before the cycle(s) involving the agents in $N_{2}$. Now fix a house $a^{*} \in F_{t}(R)$ that is being matched to an agent in $N_{1}$ according to $f^{\Gamma}(R)$ and construct a preference profile $\widetilde{R}$ such that $\widetilde{R}_{i}=R_{i}$ for $i \notin N_{2}$ and $a^{*} \widetilde{R}_{i} a$ for all $i \in N_{2}$ and all houses $a \neq a^{*}$ and $a \widetilde{R}_{i} b \Leftrightarrow a R_{i} b$ for all houses $a, b \neq a^{*}$ (meaning the only difference between $R$ and $\widetilde{R}$ is that the agents in $N_{2}$ rank $a^{*}$ at the top under $\widetilde{R}$ ). Observe that for all stages $z<t$ the mechanism $\Gamma$ induces the exact same trading cycles under $R$ and under $\widetilde{R}$. At stage $t$ only the agents in $N_{1}$ form top trading cycles: $N_{1}=W_{t}(\widetilde{R})$ and $f_{N_{1}}^{\Gamma}(R)=f_{N_{1}}^{\Gamma}(\widetilde{R})$. The agents in $N_{2}$ form top trading cycles in the next stage: $N_{2} \subset W_{t+1}(\widetilde{R})$ and $f_{N_{2}}^{\Gamma}(R)=f_{N_{2}}^{\Gamma}(\widetilde{R})$. However, by the assumption that the elimination of top trading cycles matters, the matches to agents that have not yet left the mechanism $N^{*}=N \backslash\left(\bigcup_{z=1}^{t} W_{t}(R)\right)$ must change, that is, $f_{N^{*}}^{\Gamma}(R) \neq f_{N^{*}}^{\Gamma}(\widetilde{R})$. This contradicts Lemma 1, which shows that hierarchical exchange rules must be group-nonbossy.

We are no ready to prove Theorem 2 :

Proof Start of the Induction:

$|N|=2$. Note that we must have $x_{1}=\operatorname{top}\left(R_{1}\right)$ or $x_{2}=\operatorname{top}\left(R_{2}\right)$ for $x$ to be Pareto-optimal. Choose $p$ such that an agent $i$ who is matched $\operatorname{top}\left(R_{i}\right)$ according to $x$ is the initial owner of $\operatorname{top}\left(R_{i}\right)$. He forms a trading cycle of length one pointing to $\operatorname{top}\left(R_{i}\right)$, the other agent obtains his most preferred house out of the reminder, so $x$ is the outcome of $p(\Gamma)$.

Step of the Induction:

Suppose the claim holds for $|N|=m$.

Now take a housing problem with $|N|=m+1$. 
Fix a Pareto-optimal allocation $x$. Assume w.l.o.g. that $\operatorname{top}\left(R_{1}\right)=x_{1}=$ $a$. Let $j$ be the initial owner of $a$ and consider the permutation $p$ with $p(1)=j, p(j)=1$ and $p(i)=i$ for $i \neq 1, j$. Now observe that - restricted to $K \backslash\{a\}$ and $N \backslash\{1\}-x^{\prime}=\left(x_{2}, \cdots, x_{n}\right)$ is also a Pareto-optimal allocation. By the hypothesis of the induction we can find a permutation $p^{\prime}$ on the set of roles $N \backslash\{j\}$ such that for submechanism $\Gamma^{\prime}$, that arises when eliminating agent 1 and house $a$ in first round from $p(\Gamma)$, the outcome of $p^{\prime} \Gamma^{\prime}$ is $x^{\prime}$ when presuming that agent $j$ assumed the role of agent 1 before the permutation $p^{\prime}$. Define a permutation $\bar{p}: N \rightarrow N$ such that $\bar{p}(j)=j$ and $\bar{p}(i)=p^{\prime}(i)$ otherwise. Now consider the mechanism $(\bar{p} \circ p)(\Gamma)$. According to $(\bar{p} \circ p)(\Gamma)$, agent 1 is the initial owner of house $a$, since $\operatorname{top}\left(R_{1}\right)=x_{1}=a$ agent 1 forms a top trading cycles by pointing to his own house $a$. By Lemma 2, this cycle can be eliminated, before any other possibly existing cycles without any change of the outcome of the hierarchical exchange rule. Once this cycle is eliminated we are left with the (sub)mechanism $p^{\prime} \Gamma^{\prime}$, which according to its definition, yields the outcome $\left(x_{2}, \cdots, x_{n}\right)$ for the remainder of the agents.

The theorem is an extension of the Second Welfare Theorem to an environment with indivisible goods such that each agent should be matched to at most one object. It says that, for any system of property rights and any Pareto optimum, there exists an allocation of these property rights to agents, such that the given Pareto optimum is the outcome of free trade among the agents. The definition of property rights used in this note is very general. It requires that each house is always owned by someone. It solves the problem that agents might own multiple houses by the assumption of fixed inheritance processes that prescribe the next owner of a house when the current owner leaves the market with a house. Papai (2000)'s hierarchical exchange mechanisms arguably describe the most general class of allocation mechanisms for housing problems that can be considered as derived from the assignment of property rights together with free trade.

In the literature, large sets of subclasses of hierarchical exchange mechanisms have been characterized by Ma (1994), Svensson (1999), Ergin (2000), Miyagawa (2002), Ehlers et al. (2002), Ehlers and Klaus (2004), Kesten (2009), Sonmez and Unver (2006), Ehlers and Klaus (2007), and Velez (2008). 
So the result presented here of course extends to all these. Serial dictatorships and the top trading cycles mechanism are also cases of hierarchical exchange mechanisms. Restricted to these two special cases, the Theorem of the present note has been shown by Abdulkadiroglu and Sonmez (1998).

Pycia and Unver (2009) characterize a superclass of hierarchical exchange mechanisms. They drop the requirement of reallocation-proofness from Papai (2000)'s theorem. The class they obtain differs from hierarchical exchange mechanism in that agents now cannot only be owners of houses, but also "brokers". These brokers also have control rights over houses; however, these control rights do not include the right to appropriate a house. Pycia and Unver (2009) clearly delineate a difference between ownership and brokerage of a house; it seems appropriate not to consider this superclass of hierarchical exchange mechanisms as mechanisms that can be considered to be derive from solely from the assignment of ownership rights and free trade.

\section{References}

Atila Abdulkadiroglu and Tayfun Sonmez. Random Serial Dictatorship and the Core from Random Endowments in House Allocation Problems. Econometrica, 66(3):689-701, May 1998.

Lars Ehlers and Bettina Klaus. Resource monotonicity for house allocation problems. International Journal of Game Theory, 32:545-560, 2004.

Lars Ehlers and Bettina Klaus. Consistent house allocation. Economic Theory, 30:260-274, 2007.

Lars Ehlers, Bettina Klaus, and Szilvia Papai. Strategy-proofness and population monotonicity in house allocation problems. Journal of Mathematical Economics, 38:329-339, 2002.

Haluk I. Ergin. Consistency in house allocation problems. Journal of Mathematical Economics, 34:77-97, 2000.

Onur Kesten. Coalitional strategy-proofness and resource monotonicity for 
house allocation problems. International Journal of Game Theory, 38: 17-21, 2009.

Jinpeng Ma. Strategy-proofness and strict core in a market with indivisibilities. International Journal of Game Theory, 23:75-83, 1994.

Eiichi Miyagawa. Strategy-proofness and the core in house allocation problems. Games and Economic Behavior, 38:347-361, 2002.

Szilvia Papai. Strategyproof Assignment by Hierachical Exchange. Econometrica, 68(6):1403-1433, November 2000.

Marek Pycia and Utku Unver. A Theory of House Allocation and Exchange Mechanisms, January 2009. available at SSRN: http://ssrn.com/abstract $=1079505$.

Tayfun Sonmez and Utku Unver. Kidney exchange with good Samaritian donors: A characterization. Working paper, Boston College and University of Pittsburgh, 2006.

Lars-Gunnar Svensson. Strategy-proof allocation of indivisible goods. Social Choice and Welfare, 16:557-567, 1999.

Rodrigo A. Velez. Revisiting Consistency in House Allocation Problems. Working paper, 2008. 